\title{
Cardiovascular involvement in patients with 2019 novel coronavirus disease
}

\author{
Chenze $\mathrm{Li}^{1}$, Dao Wen Wang ${ }^{2}$, Chunxia Zhao ${ }^{2}$ \\ 'Department of Cardiology, Zhongnan Hospital of Wuhan University, Wuhan, Hubei Province, China \\ ${ }^{2}$ Division of Cardiology, Department of Internal Medicine and Hubei Key Laboratory of Genetics and Molecular \\ Mechanisms of Cardiological Disorders, Tongji Hospital, Tongji Medical College, Huazhong University of \\ Science and Technology, Wuhan 430030, Hubei Province, China
}

Address for Correspondence: Dr. Chunxia Zhao, MD, PhD, Division of Cardiology, Department of Internal Medicine, Tongii Hospital, Tongii Medical College, Huazhong University of Science and Technology, Wuhan 430030, Hubei Province, China.

E-mail: zhaocx2001@126.com

\begin{tabular}{|l|}
\hline Access this article online \\
Website: \\
www.intern-med.com \\
\hline DOI: \\
10.2478/jtim-2021-0016 \\
\hline Quick Response Code: \\
\hline
\end{tabular}

\section{ABSTRACT}

2019 novel coronavirus disease (COVID-19) is caused by the infection of severe acute respiratory syndrome novel coronavirus (SARS-CoV-2). It is characterized by substantial respiratory symptoms and complicated with widespread other organ injuries. Cardiovascular impairment is one of the notable extrapulmonary manifestations, in terms of the deterioration of pre-existing cardiovascular diseases and newly onset acute events. We hereby review the high-quality reports about cardiovascular involvement in COVID-19 and summarize the main clinical characteristics of cardiac relevance for the all the first line clinical physicians. Additionally, the possible underlying mechanisms and the rationale for the application of specific medications, such as renin-angiotensin-aldosterone system inhibitors and hydroxychloroquine are also discussed.

Key words: 2019 novel coronavirus disease, cardiovascular system, injuries

\section{INTRODUCTION}

In late December 2019, a novel virus, named as severe acute respiratory syndrome novel coronavirus (SARS-CoV-2), caused a worldwide pandemic that has affect billions of lives. ${ }^{[1-3]}$. Until now, more than $86,000,000$ individuals have been confirmed as 2019 novel coronavirus disease (COVID-19) patients as of January 06, 2021, and this virus has contributed to around $3 \%$ of deaths (https://www.cdc.gov). Compared with other coronaviruses, such as severe acute respiratory syndrome (SARS) and Middle East Respiratory Coronavirus (MERS), COVID-19 seems to have lower mortality, but its basic reproduction number is up to 2.0-2.5. ${ }^{[4]}$ Based on the existing knowledge, it is not only a pneumonia, but actually is systemic disease, and the heart appears to be one of the most frequently and critically injured extrapulmonary organs. ${ }^{[5,6]}$ Patients who were older than 60 years old and complicated with more cardiovascular commodities had a higher fatality risk than young patients with less cardiovascular disease. ${ }^{[7]}$ Currently, studies on the association of the cardiovascular system with COVID-19 are rapidly increasing, ${ }^{[8-11]}$ while a comprehensive review of the recent advance is limited. The spread of SARS-CoV-2 was still not eliminated and may cause another large-scale explosion. Therefore, a better understanding of the underlying mechanism of cardiovascular complications and clinical manifestation of COVID-19 is still needed. In the current review, we intended to discuss the latest findings of cardiovascular involvement in COVID-19 patients and its possible mechanism.

\section{THE BIOLOGICAL CHARACTERISTICS OF SARS-COV-2 AND ITS THERAPEUTIC TARGETS}

Since the outbreak of COVID-19, the sequence of SARS-CoV-2 has been decoded by whole genomic sequencing, and the viral protein structure has been well analyzed. ${ }^{[12,13]}$ SARS-CoV-2 is a positive-sense and singlestranded enveloped RNA virus, belonging 
to beta-coronaviruses. ${ }^{[14]}$ The length of the genome of SARS-Cov-2 is approximately $30 \mathrm{~kb}$ and contains $24-27$ coding genes. ${ }^{[15]}$ It has a crown-like structure and consists of four structural proteins, including spike (S), envelope (E), membrane $(\mathrm{M})$, and nucleocapsid $(\mathrm{N})$. Among them, the $\mathrm{N}$ protein surrounded the viral RNA and the $\mathrm{S}$ protein is a crucial structural protein that can attach the angiotensinconverting enzyme 2 (ACE2) receptor. After the virus enters the human body, dominantly via respiratory tract inspiration, the subunit $\mathrm{S} 1$ of the $\mathrm{S}$ protein binds to its receptor ACE2, and then the S protein was primed by the transmembrane serine protease 2 (TMPRSS2); thereafter, the viral membrane is fused with the host cell's membrane to enter the cytoplasm and thus finish invasion process into the host cell. ${ }^{[16]}$ The high expression of ACE2 in tissue was considered one of the main reasons for tissue-specific injury. ${ }^{[17]}$ Previous studies have shown that ACE2 is highly expressed in heart tissues, especially in pericyte cells. ${ }^{[18]}$ Of note, in animal models, the administration of the renin-angiotensin-aldosterone system (RAAS) inhibitors could upregulate the expression of ACE2. ${ }^{[19]}$ However, RAAS inhibitors, including angiotensin-converting enzyme inhibitor (ACEI) and angiotensin II receptor blockers $\left(\mathrm{ARB}_{\mathrm{S}}\right)$, are frequently used to treat patients with hypertension, coronary heart diseases, and heart failure. There is a wide concern about the bad or benefit of RAAS inhibitors in COVID-19 patients. A comprehensive review of the association of RAAS inhibitors on the pathophysiology of COVID-19 has been well discussed elsewhere. ${ }^{[20]}$ Taken together, a beneficial role of RAAS inhibitors on COVID-19 patients can be observed in most studies. Especially, a recent study conducted by Chen et al. investigated the association of the mortality with the pre-hospitalization use of RAAS inhibitors in COVID-19 patients. ${ }^{[21]}$ They revealed that COVID-19 patients could benefit from the pre-hospitalization use of RAAS inhibitors, and the mechanism underlying this benefit may come from the reduced inflammatory response after the administration of RAAS inhibitors in patients with COVID-19.

Besides, the S protein of SARS-CoV-2 also can be primed in endosomal by the endosomal cathepsin $\mathrm{L}$ and cysteine proteases cathepsin $\mathrm{B}$, and then enters into the cytoplasm. Researchers have reported that the endosomal pathway can be inhibited by hydroxychloroquine, a lysosomotropic agent. ${ }^{[22,23]}$ In an early study, ${ }^{[24]}$ investigators use hydroxychloroquine combined with azithromycin to test its efficiency in decreasing the viral load of SARSCoV-2. Surprisingly, the SARS-CoV-2 was significantly inhibited after hydroxychloroquine application. Also, a retrospective study conducted by $\mathrm{Yu}$ et al. found that the application of hydroxychloroquine can effectively lower the in-hospital mortality of COVID-19 patients, compared with those without hydroxychloroquine treatment during hospitalization. ${ }^{[25]}$ Moreover, in COVID-19 patients with hydroxychloroquine treatment, their biomarker levels of organ injury, such as liver (albumin), heart (high-sensitivity cardiac troponin I, hs-cTnI), inflammation (interleukin-6, IL-6) are lower than those without hydroxychloroquine treatment. ${ }^{[26]}$ Likewise, Arshad et al. reported that COVID-19 patients in hydroxychloroquine-treated patients had lower mortality than those in non-hydroxychloroquine group. ${ }^{[27]}$ However, other studies showed that there is no significant difference in the rate of in-hospital death between the hydroxychloroquine treatment group and no hydroxychloroquine treatment group. ${ }^{[28,29]}$ The reason for these may be that in different studies, the dose and the duration of the application of hydroxychloroquine in treating patients are diverse.

\section{PRE-CARDIOVASCULAR DISEASES IN COVID-19 PATIENTS}

COVID-19 patients were often complicated with cardiovascular comorbidities, such as hypertension, coronary heart disease, and diabetes. ${ }^{[30-33]}$ The studies describing the baseline characteristics of COVID-19 patients has shown similar features. An early description of 138 COVID-19 patients revealed that $14.5 \%$ of patients had cardiovascular commodities. ${ }^{[31]}$ The most common cardiovascular disease is hypertension with approximately $31.2 \%$ of patients. Moreover, a higher prevalence of cardiovascular disease is associated with a higher risk of progressing into critically ill. Similarly, the high prevalence of cardiovascular comorbidities was confirmed in the study by our team. ${ }^{[34]}$ We demonstrate that the rate of presenting hypertension is $34.2 \%$, coronary heart disease is $8.8 \%$, and diabetes is $14.1 \%$. Also, patients who had cardiovascular complications are more likely to progress into critically ill status. When patients were divided into three groups, the patients in the critically ill group had a high incidence of cardiovascular disease. However, compared with critically ill survivors, critically ill deaths did not show a high prevalence of hypertension, coronary heart disease, and diabetes. Another multicenter study included 1,099 hospitalized COVID-19 patients indicated that the fatality rate of patients with cardiovascular commodities was $17.6 \%{ }^{[35]}$ Of these, $15.0 \%$ patients has hypertension, $7.4 \%$ diabetes, and $2.5 \%$ coronary heart disease. Similar features of cardiovascular comorbidities in COVID-19 patients were also observed in other countries, except for China. ${ }^{[36-38]}$ A retrospective study involving 393 Caucasian patients with COVID-19 reported that $50.1 \%$ of patients had a history of hypertension, $25.2 \%$ had a history of diabetes, and $13.7 \%$ had a history of coronary heart disease. ${ }^{[36]}$ Also, in an Italy study report, the prevalence of cardiovascular 


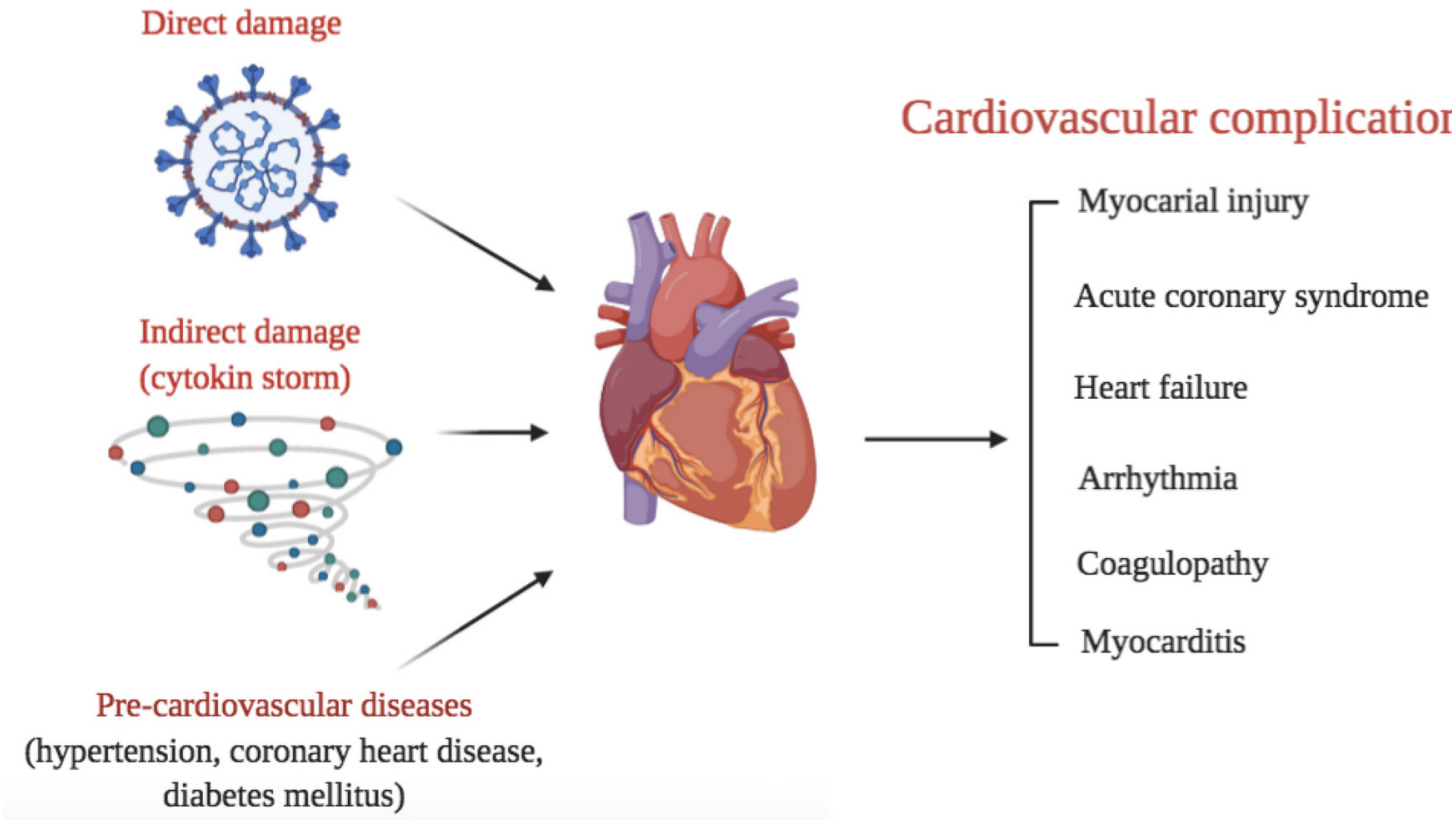

Figure 1: Cardiovascular involvements during the infection of SARS-CoV-2 caused by direct damage, indirect damage, and aggravated by the existence of pre-cardiovascular diseases

comorbidities showed a similar trend. ${ }^{[39]} \mathrm{Up}$ to $49.0 \%$ of patients had hypertension, $17.0 \%$ of patients had diabetes, $18.0 \%$ of patients had hypercholesterolemia, and an overall $2.1 \%$ of patients had cardiomyopathy/heart failure. Thus, comorbidities of cardiovascular diseases are very common and leads to high risk of critical illness and death.

\section{CARDIOVASCULAR COMPLICATIONS IN COVID-19 PATIENTS}

The predominant clinical presentation of COVID-19 patients is bilateral pneumonia, but other cardiovascular complications, including myocardial injury, heart failure, arrhythmia, acute coronary syndrome (ACS), thrombus or coagulopathy, can also occur in COVID-19 patients (Figure 1). Furthermore, cardiovascular involvements are very common seen in COVID-19 patients (Table 1).

\section{Myocardial injury}

In most of the studies, the degree of myocardial injury was evaluated by the level of hs-cTnI. ${ }^{[40,41]}$ The levels of hs-cTnI have been confirmed as an independent risk factor of poor prognosis in COVID-19 patients. ${ }^{[11]}$ In a report involving 671 severe COVID-19 patients, $15.8 \%$ of patients were found to have a myocardial injury. ${ }^{[10]}$ A subsequent study of 311 patients showed that myocardial injury was associated with a 17.66-fold increase of in-hospital mortality. In another report of 2068 COVID-19 patients in Wuhan, a total of 181 patients $(8.8 \%)$ had elevated hs-c'TnI levels. ${ }^{[34]}$ Compared with critically ill patients, non-critically ill patients had a lower level of hs-c'TnI and lower incidence of hs-cTnI elevation. In non-critically ill patients, only $2.3 \%$ of patients were presented with a high hs-cTnI level. For most of non-critically ill patients, their myocardial biomarkers, including hs-cTnI, creatine kinase-MB (CK-MB) and NT-ProBNP, were within a normal range. However, in critically ill patients, the levels of myocardial biomarkers are significantly elevated. For critically ill patients, $30.3 \%$ of patients had elevated hs-c'TnI level on admission, $93.8 \%$ of patients had elevated NT-proBNP level on admission, $11.1 \%$ of patients had elevated CK-MB level on admission, and $39.2 \%$ of patients had elevated myoglobin $(\mathrm{Mb})$ level on admission. Compared with critically ill survivors, critically ill deaths had a higher level of hs-cTnI level at admission and throughout hospitalization. Moreover, the level of hs-cTnI and other myocardial biomarkers decreased on the third day after admission in critically ill survivors. Among critically ill deaths, the levels of myocardial biomarkers, especially for hs-cTnI level, continuously increase during the whole hospitalization. In this study, one per standard deviation (SD) increase of hs-c'TnI on admission was associated with 1.31-fold risk of in-hospital mortality. After adjusted for baseline characteristics, the level of hs-cTnI was still independently associated with increased in-hospital mortality.

\section{Acute coronary syndrome}

In most of the COVID-19 patients, they were presented with fever, dyspnea, and diarrhea on admission, but some 
Table 1: Cardiovascular involvements in patients with COVD-19

\begin{tabular}{|c|c|c|c|c|c|}
\hline & Reference & Race & Study participants & No. of patients & Prevalence \\
\hline \multicolumn{6}{|l|}{ Pre-cardiovascular diseases } \\
\hline \multirow[t]{5}{*}{ Hypertension } & 31 & Chinese & COVID-19 & 138 & $31.2 \%$ \\
\hline & 34 & Chinese & COVID-19 & 2068 & $34.2 \%$ \\
\hline & 35 & Chinese & COVID-19 & 1,099 & $15.0 \%$ \\
\hline & 36 & Caucasian & COVID-19 & 393 & $50.1 \%$ \\
\hline & 39 & Caucasian & COVID-19 & 1,591 & $49.0 \%$ \\
\hline \multirow[t]{3}{*}{ Coronary heart disease } & 34 & Chinese & COVID-19 & 2,068 & $8.8 \%$ \\
\hline & 35 & Chinese & COVID-19 & 1,099 & $2.5 \%$ \\
\hline & 36 & Caucasian & COVID-19 & 393 & $13.7 \%$ \\
\hline \multirow[t]{4}{*}{ Diabetes } & 34 & Chinese & COVID-19 & 2,068 & $14.1 \%$ \\
\hline & 35 & Chinese & COVID-19 & 1,099 & $7.4 \%$ \\
\hline & 36 & Caucasian & COVID-19 & 393 & $25.2 \%$ \\
\hline & 39 & Caucasian & COVID-19 & 1,591 & $17.0 \%$ \\
\hline \multicolumn{6}{|l|}{ Cardiovascular complications } \\
\hline \multirow[t]{2}{*}{ Myocardial injury } & 10 & Chinese & COVID-19 & 671 & $15.8 \%$ \\
\hline & 34 & Chinese & COVID-19 & 2,068 & $8.8 \%$ \\
\hline \multirow[t]{2}{*}{ Acute coronary syndrome } & 42 & Caucasian & COVID-19 with ST-segment elevation & 28 & $85.7 \%$ \\
\hline & 34 & Chinese & COVID-19 & 2,068 & $0.2 \%$ \\
\hline \multirow[t]{3}{*}{ Heart failure } & 45 & Chinese & COVID-19 & 799 & $49.0 \%$ \\
\hline & 45 & Chinese & Died COVID-19 & 113 & $85.0 \%$ \\
\hline & 34 & Chinese & COVID-19 & 2,068 & $64.3 \%$ \\
\hline \multirow[t]{5}{*}{ Arrhythmia } & 31 & Chinese & COVID-19 & 138 & $16.7 \%$ \\
\hline & 31 & Chinese & COVID-19 in intensive care unit & 36 & $44.4 \%$ \\
\hline & 31 & Chinese & COVID-19 in non-intensive care unit & 102 & $6.9 \%$ \\
\hline & 34 & Chinese & COVID-19 with elevated hs-cTnl & 227 & $47.1 \%$ \\
\hline & 34 & Chinese & COVID-19 without elevated hs-cTnl & 249 & $12.4 \%$ \\
\hline \multirow[t]{3}{*}{ Coagulopathy } & 35 & Chinese & COVID-19 & 1,099 & $46.4 \%$ \\
\hline & 7 & Chinese & COVID-19 & 191 & $42.0 \%$ \\
\hline & 34 & Chinese & COVID-19 & 2,068 & $58.6 \%$ \\
\hline Myocarditis & - & - & - & - & - \\
\hline
\end{tabular}

COVID-19: 2019 novel coronavirus disease; hs-cTnl: high sensitivity cardiac troponin I.

patients did not have the above symptoms when they were admitted to the hospital. For those without typical symptoms, chest pain is one of the initial symptoms. A report from Italy involving 28 COVID-19 patients with ST-segment elevation showed that 17 of patients need revascularization. More importantly, in $85.7 \%$ of patients (24/28), the first clinical symptoms was chest pain. ${ }^{[42]}$ In the study from Li et al., 3.1\% of patients were presented with chest pain, although the incidence of chest pain in non-critically ill patients was not different from critically ill patients. ${ }^{[34]}$ The comparison of the incidence of ACS between COVID-19 period and pre-COVID-19 period has shown that the rate of hospitalization for ACS and percutaneous coronary intervention decrease with a $38 \%-48 \%$ reduction in Italy. ${ }^{[43,44]}$ However, the incidence of ACS is underestimated. Due to the limited medical sources, very few patients take the examination of coronary angiography. Electrocardiogram (ECG) is, therefore, the most used technique to assess the degree of myocardial ischemia during COVID-19 outbreak. In the study from a 
Wuhan single-center, 22 patients performed ECG, and 14 of them had T-wave depression and inversion, ST-segment depression, or Q waves. ${ }^{[4]}$ In our study, the results showed that 118 patients had the findings of ECG. The elevation/ depression of ST segment accounted for $72.5 \%$. Only three patients were diagnosed with myocardial infarction by coronary angiography. ${ }^{[34]}$ The mechanism of ACS underlying COVID-19 patients is suspected to result from systemic inflammation and cytokine storm that induced plaque rupture, microthrombosis, and coronary spasm.

\section{Heart failure}

Heart failure is one of the most common causes of death in COVID-19 patients. According to a report from Tongji hospital including 799 COVID-19 patients, they observed that $49.0 \%$ patients had an elevation of NT-proBNP (the cutoff was $285 \mathrm{pg} / \mathrm{mL}$ ). ${ }^{[4]}$ In dead COVID-19 patients, this proportion increased to $85 \%$. Similarly, the high incidence of NT-proBNP elevation was confirmed in another study that enrolled 2,068 patients. ${ }^{[34]}$ In this study, more than $64.3 \%$ of patients had elevated NT-proBNP on admission (the cutoff was $62.9 \mathrm{pg} / \mathrm{mL}$ ). Especially, critically ill patients had a higher incidence of NT-proBNP elevation and NT-proBNP level, compared with non-critically ill patients. On the third day, the level and the elevated rate of NT-proBNP started to decrease to the normal range. Besides, the level of NT-proBNP is positively correlated with the level of inflammation biomarkers, such as IL-6, C-reactive protein (CRP), and interleukin-8 (IL-8), which showed an association of systemic inflammation with the incidence of heart failure. However, the application of echocardiography to evaluate the cardiac function of patients with elevated NT-proBNP is not feasible during the outbreak of COVID-19. Therefore, whether the elevation of NT-proBNP is due to heart failure or hypoxia is not easy to differentiate. However, in a case series involving 100 patients with echocardiography examination, ${ }^{[46]}$ the findings showed that 39\% patients had right ventricular dilatation and dysfunction, $16 \%$ of patients had left ventricular diastolic dysfunction, and 10\% patients had left ventricular systolic dysfunction. The reasons for the high incidence of heart failure is still unknown. However, given that most of COVID-19 patients were older than 60 years old and complicated with cardiovascular comorbidities. Heart failure may be triggered by high fever, arrhythmia, and systemic inflammation. Additionally, acute myocardial injury can exacerbate pre-existing cardiovascular disease and induce heart failure. On the other hand, due to the overactivation of the immune system, stress-induced cardiomyopathy, or cytokine storm-induced myocardial dysfunction (inflammatory cardiomyopathy), sepsis-related cardiac dysfunction are also the possible mechanisms for the incidence of heart failure or cardiac dysfunction.

\section{Arrhythmia}

Arrhythmia is a common clinical cardiovascular manifestation in COVID-19 patients. In the early report involving 138 patients, $16.7 \%$ of patients had arrhythmias. ${ }^{[31]}$ In hospitalized patients in the intensive care unit (ICU), the incidence of arrhythmias is $44.4 \%$, which was higher than non-ICU patients $(6.9 \%)$. But, the specific type of arrhythmia in this study was not pointed out. Besides, palpitation is also one of the frequent clinical presentation, except for fever and cough, although the rate of palpitation was not significantly different between critically ill patients and non-critically ill patients. ${ }^{[31]}$ Moreover, the incidence of arrhythmias is associated with the degree of myocardial injury. ${ }^{[34]}$ A study with 2,068 COVID-19 patients demonstrates that the incidence of arrhythmia is higher in COVID-19 patients with elevated hs-cTnI on admission, compared with patients without elevated hs-cTnI admission. This association was still consistent when patients were stratified according to the hs-cTnI level during hospitalization. In patients with elevated hs-cTnI during hospitalization, the rate of arrhythmia was $47.1 \%$. However, the rate of arrhythmias was $12.4 \%$ in patients without elevated hs-c'TnI during hospitalization, suggesting some cardiac injury cannot be evident by its biomarkers such as hs-cTnI and NT-ProBNP. In this study, the types of arrhythmias were specified, including ventricular tachycardia, supraventricular tachycardia, atrial fibrillation, atrial flutter, second-degree (type 2) or third-degree atrioventricular block, and sick sinus syndrome. Except of rhythm disturbance, cardiac arrest also frequently occurred in COVID-19 patients. In 2,068 patients, 77 patients had experienced cardiac arrest. Importantly, cardiac arrest occurred only in critically ill patients. In critically ill victims, $1.7 \%$ of patients was cardiac arrest survivors. For critically ill deaths, the incidence rate of cardiac arrest was significantly increased. However, the real association of arrhythmia and COVID-19 remains unclear. The etiology of arrhythmia in COVID-19 patients can be multi-factor. For example, myocardial injury, sepsis, cytokine storm, systemic inflammation, and other application of antiviral and antibiotic medications.

\section{Coagulopathy}

Coagulopathy is disorders in coagulation function. Hypercoagulation is also frequently observed in COVID-19 patients. A study reported that the elevated D-dimer level on admission was associated with a worse clinical outcome. ${ }^{[4]}$ If the cutoff value is $0.5 \mathrm{ug} / \mathrm{mL},{ }^{[35]} 46.4 \%$ of patients had elevated D-dimer level, and $42.0 \%$ of patients had elevated D-dimer level in another study if the cut-off value was set to $1.0 \mathrm{ug} / \mathrm{mL}^{7}$. In the report of Tongji hospital, critically ill patients had a higher rate of hypercoagulation, indicated by elevated $\mathrm{D}$-dimer, fibrinogen $(\mathrm{Fbg})$, and international normalized ratio (INR). In a total of 2,068 
patients, $58.6 \%$ of patients had elevated D-dimer, 58.0\% patients had elevated Fbg (the cut-off value was $4.0 \mathrm{~g} / \mathrm{L}$ ), and $10.0 \%$ patients had abnormal INR (normal range $0.8-1.2$ ). The rate of hypercoagulation was significantly increased in critically ill patients. In critically ill survivors, the median level of $\mathrm{D}$-dimer on admission was $2 \mathrm{ug} / \mathrm{mL}$, but decreased on the third day after admission. For critically ill deaths, the level of D-dimer attained to the peak on the seventh day. Except for the level of D-dimer, the level of platelet and prothrombin time are also the indicators of coagulation abnormalities. A study of 41 patients with COVID-19 from Wuhan observed a reduction of platelet counts and prolongation of prothrombin time, although the changes were modest. ${ }^{[30]}$ In this report, the rate of a low platelet count was $5 \%$, and the time of prothrombin time was $11.1 \mathrm{~s}$ in non-ICU patients; by contrast, the time of prothrombin time was 11.2 non-ICU patients. Consistently, another retrospective research studying liver dysfunction in COVID-19 patients also observed a modest but significant prolongation of prothrombin time. ${ }^{[48]}$ Thus, these retrospective data showed that hypercoagulation and also thrombosis, including microthrombosis, is a common complication in COVID-19 patients. Importantly, the level of D-dimer is independently associated with increased in-hospital mortality. ${ }^{[34]}$ The findings showed that 1 per SD increase in the level of D-dimer on admission 1.48fold increase in the risk for in-hospital death, and 1 per $\mathrm{SD}$ increase in the level of $\mathrm{D}$-dimer on the third day after admission was associated with 2.71 hazard ratio for inhospital mortality, and 1 per SD increase in the level of D-dimer on the $>14$ th day after admission was associated with a 3.05-fold risk for in-hospital mortality. ${ }^{[34]}$ When the dead patients were divided into two groups: deaths less than 7 days and deaths more than 7 days, similar observations were found that those who died within 7 days had a higher level of D-dimer, Fbg, and INR than those who died after 7 days. These suggested that the hypercoagulation status promoted the progression of COVID-19. For the investigation of the mechanism of hypercoagulation is still limited. However, in the study from Wuhan assessing the longitude dynamic changes of inflammation biomarkers and coagulation biomarkers, we found significant positive correlations of the level D-dimer, Fbg, INR, and the level of IL-6, CRP, which partially implied a systemic inflammation-inducing the hypercoagulation status. Another hypothesis is that some medications, such as antiviral drug, can arise hypercoagulation status.

Besides, venous thromboembolism was also commonly observed in COVID-19 patients. ${ }^{[49]}$ A report studying COVID-19 patients by echocardiography showed that deep vein thrombosis and pulmonary embolism were found in COVID-19 patients. ${ }^{[50,51]}$ An autopsy study involving 12 patients revealed that seven of patients had deep vein thrombosis, and four of patients had a pulmonary embolism, but they were all not suspected as patients with venous thromboembolism before death. ${ }^{[49,52,53]}$ These implied that venous thromboembolism is a pivotal factor contributing to the death in COVID-19 patients, but it is not easy to be identified during the infection of COVID-19.

Given that most of COVID-19 patients were under hypercoagulation status. The efficiency of the anticoagulation drugs in treating COVID-19 patients is of interest to investigators. A retrospective study of 449 COVID-19 patients showed a reduction of in-hospital mortality after application of prophylactic heparin. ${ }^{[54]}$ The in-hospital mortality was $40.0 \%$ in patients with prophylactic heparin treatment group with sepsis-induced coagulopathy score $\geq 4$, which was significantly lower than the mortality in patients without the prophylactic heparin treatment group with $\geq 4(64.2 \% ; \mathrm{p}=0.029)$. However, in another study involving 2,773 patients, $28 \%$ of patients were treated with the anticoagulation drug. ${ }^{[5]}$ The overall in-hospital mortality was not significantly different between those treated with the anticoagulation drug and those without the anticoagulation drug. But, a prolongation of median survival time was observed in patients treated with the anticoagulation drug. The survival time was 21 days in patients with treatment of anticoagulation drug, which significantly longer than 14 days in patients without anticoagulation drug treatment. In the study involving 2,068 COVID-19 patients, ${ }^{[34]} 16.8 \%$ of patients received anticoagulation treatment during hospitalization. Of them, critically ill patients were more often treated with the anticoagulation drug than noncritically ill patients. However, among critically ill patients, the proportion of application of anticoagulation drug was $53.6 \%$ in critically ill survivors and $55.2 \%$ in critically ill deaths. No significant efficiency of the application of anticoagulation drugs on in-hospital mortality was observed in critically ill patients. In this study, the type of anticoagulation drug includes dabigatran, heparin, warfarin, rivaroxaban, and sulodexide. However, the potential confounding factors and the indications of the anticoagulation drug were not clarified in these studies. In future studies, the dose, the administration time, and the type of anticoagulation drug should be analyzed. Alternatively, prospective, double, randomized trials are encouraged to prospectively evaluate the efficiency of the anticoagulation drug in COVID-19 patients.

\section{Myocarditis}

Whether patients with elevated hs-cTnI level results from myocarditis is unclear. Given that few COVID-19 patients received echocardiography or magnetic resonance imaging (MRI) examination during the outbreak of COVID-19. According to the data from a retrospective study involving 112 COVID-19 patients, $37.5 \%$ of patients had an elevation 
of hs-cTnI, but there were no significant abnormalities in segmental wall motion and left ventricular ejection fraction on echocardiography. ${ }^{[56]}$ In the study of 2,068 COVID-19 patients from Wuhan, ${ }^{[34]} 49$ of patients were examined by echocardiography. In COVID-19 patients, $10.2 \%$ of patients had reduced left ventricular ejection fraction $(\mathrm{LVEF}<40 \%)$, and $25.8 \%$ of patients had pericardial effusion. As a limited sample size, the comparisons between groups cannot be achieved. However, this evidence suggests a nature that myocardial injury may mostly result from systemic inflammation rather than the direct injury of SARSCoV-2. Nevertheless, some sporadic cases reported that COVID-19 patients had typical symptoms of myocarditis. In a case report, ${ }^{[57]}$ a 53 -year-old female admitted to the hospital presented with elevated myocardial biomarkers and elevated ST segment on the ECG. Echocardiography showed a significant reduction in LVEF. MRI thereafter also confirmed the diagnosis of acute myocarditis. A similar case was also reported by another team. ${ }^{[58]}$ They successfully saved COVID-19 patients with fulminant myocarditis by applying methylprednisolone. But, the supportive evidence for direct myocardial injury from histology is still limited. In an autopay of COVID-19 patients, there was no obvious evidence showing a direct impairment on heart, although this patient died because of cardiac arrest. ${ }^{[59]}$ But investigators found coronavirus particles and viral RNA from another endomyocardial biopsy of COVID-19 patients. ${ }^{[00]}$ Overall, there is no strong evidence support that myocarditis is the main consequence of the direct myocardial injury of SARS-CoV-2. It is more likely to be a secondary consequence of systemic inflammation.

\section{OTHER RELATED VASCULAR DISEASES}

For COVID-19 patients, they were also complicated with other vascular diseases, such as diabetes mellitus and kidney diseases. In a recent report conducted by $\mathrm{Yu}$ et al., the researchers showed that about $20.8 \%$ hospitalized patients with COVID-19 had a history of diabetes mellitus. ${ }^{[61]}$ Of the all T2DM patients, 229/689 (33.2\%) patients were newly diagnosed. Among patients with diabetes mellitus, the levels of fasting glucose ranging from 3.9 to 10.0 $\mathrm{mmol} / \mathrm{L}$ were associated with lower in-hospital mortality. ${ }^{[62]}$ Furthermore, increasing evidence suggested that the use of metformin did not increase the rate of death. ${ }^{[63]}$ However, the application of insulin might worse the clinical outcome, and the mechanism linking this association may arise from the aggravation of cytokines storm. ${ }^{[61]}$ Accordingly, patients with COVID-19 and diabetes mellitus should be treated with insulin with caution. Nevertheless, the optimal management in COVID-19 patients with diabetes mellitus is needed to be further investigated.
On the other hand, although the prevalence of prior kidney disease in COVID-19 patients is rare, the incident acute kidney injuries are not uncommon. A pooled study indicated that the incidence of acute kidney injuries in all COVID-19 patients was $6.5 \%$, and this rate was higher in critically ill patients $(32.5 \%)$. ${ }^{[64]}$ Because ACE2 receptors are not only widely expressed in the respiratory tract but also in other epithelial cells, such as epithelial apical brush borders of the proximal renal tubules and podocytes, SARS-CoV-2 could utilize ACE2 to enter into the cells, resulting in the occurrence of renal injury. ${ }^{[65]}$ Meanwhile, the systemic cytokine storm, ischemia, and other risk factors are also responsible for the pathogenesis of acute kidney disease. ${ }^{[6]}$ More attention on short- and long-term renal recovery associated with acute kidney disease is needed.

\section{CONCLUSIONS}

The cardiovascular manifestation caused by COVID-19 has been appreciated, except for the pulmonary complications of SARS-CoV-2. COVID-19 patients with pre-existing cardiovascular disease or new-onset myocardial injury had a poor prognosis. Therefore, monitoring the cardiovascular system, including the measurement of biomarkers of myocardial injury and cardiac function (hs-cTnI and NT-ProBNP), electrocardiograph, and echocardiography or MRI during SARS-CoV-2 infection is of importance. Deep analysis of echocardiography will more sensitively find cardiac injury. ${ }^{[52,67]}$ More importantly, it is urgent for scientists to identify more cardioprotective drugs, including RAAS inhibitors, $\beta$-receptor blockade, and statins ${ }^{[21,34,68]}$ in treating COVID-19 patients in the future.

\section{Conflict of interest}

None.

\section{REFERENCES}

1. Zhu N, Zhang D, Wang W, Li X, Yang B, Song J, et al. A Novel Coronavirus from Patients with Pneumonia in China, 2019. N Engl J Med 2020;382:727-733.

2. Zhou P, Yang XL, Wang XG, Hu B, Zhang L, Zhang W, et al. A pneumonia outbreak associated with a new coronavirus of probable bat origin. Nature 2020;579:270-3.

3. Lu R, Zhao X, Li J, Niu P, Yang B, Wu H, et al. Genomic characterisation and epidemiology of 2019 novel coronavirus: implications for virus origins and receptor binding. Lancet 2020;395:565-74.

4. Callaway E, Cyranoski D, Mallapaty S, Stoye E, Tollefson J. The coronavirus pandemic in five powerful charts. Nature 2020;579:482-3.

5. Driggin E, Madhavan MV, Bikdeli B, Chuich T, Laracy J, Biondi-Zoccai G, et al. Cardiovascular Considerations for Patients, Health Care Workers, and Health Systems During the COVID-19 Pandemic. J Am Coll Cardiol 2020;75:2352-71.

6. Clerkin KJ, Fried JA, Raikhelkar J, Sayer G, Griffin JM, Masoumi A, et al. COVID-19 and Cardiovascular Disease. Circulation 2020;141:1648-55. 
7. Zhou F, Yu T, Du R, Fan G, Liu Y, Liu Z, et al. Clinical course and risk factors for mortality of adult inpatients with COVID-19 in Wuhan, China: a retrospective cohort study. Lancet 2020;395:1054-62.

8. Knight DS, Kotecha T, Razvi Y, Chacko L, Brown JT, Jeetley PS, et al. COVID-19: Myocardial injury in survivors. Circulation 2020; 142:1120-2.

9. Tucker NR, Chaffin M, Bedi KC, Papangeli I, Akkad AD, Arduini A, et al. Myocyte-Specific Upregulation of ACE2 in Cardiovascular Disease: Implications for SARS-CoV-2-Mediated Myocarditis. Circulation 2020;142:708-10.

10. Shi S, Qin M, Cai Y, Liu T, Shen B, Yang F, et al. Characteristics and clinical significance of myocardial injury in patients with severe coronavirus disease 2019. Eur Heart J 2020;41:2070-79.

11. Nie SF, Yu M, Xie T, Yang F, Wang HB, Wang ZH, et al. Cardiac Troponin I Is an Independent Predictor for Mortality in Hospitalized Patients With COVID-19. Circulation 2020;142:608-10.

12. Wrapp D, Wang N, Corbett KS, Goldsmith JA, Hsieh CL, Abiona O, et al. Cryo-EM structure of the 2019-nCoV spike in the prefusion conformation. Science 2020;367:1260-63.

13. Walls AC, Park YJ, Tortorici MA, Wall A, McGuire AT and Veesler D. Structure, Function, and Antigenicity of the SARS-CoV-2 Spike Glycoprotein. Cell 2020;181:281-292.e6.

14. Adachi S, Koma T, Doi N, Nomaguchi M and Adachi A. Commentary: Origin and evolution of pathogenic coronaviruses. Front Immunol 2020;11:811.

15. Wu F, Zhao S, Yu B, Chen YM, Wang W, Song ZG, et al. A new coronavirus associated with human respiratory disease in China. Nature 2020;579:265-69.

16. Hoffmann M, Kleine-Weber H, Schroeder S, Krüger N, Herrler T, Erichsen S, et al. SARS-CoV-2 Cell Entry Depends on ACE2 and TMPRSS2 and Is Blocked by a Clinically Proven Protease Inhibitor. Cell 2020;181:271-280.e8.

17. Gupta A, Madhavan MV, Sehgal K, Nair N, Mahajan S, Sehrawat TS, et al. Extrapulmonary manifestations of COVID-19. Nat Med 2020;26:1017-32.

18. Chen L, Li X, Chen M, Feng Y and Xiong C. The ACE2 expression in human heart indicates new potential mechanism of heart injury among patients infected with SARS-CoV-2. Cardiovasc Res 2020;116:1097-100.

19. Ferrario CM, Jessup J, Chappell MC, Averill DB, Brosnihan KB, Tallant EA, et al. Effect of angiotensin-converting enzyme inhibition and angiotensin II receptor blockers on cardiac angiotensin-converting enzyme 2. Circulation 2005;111:2605-10.

20. Wang JJ, Edin ML, Zeldin DC, Li C, Wang DW and Chen C. Good or bad: Application of RAAS inhibitors in COVID-19 patients with cardiovascular comorbidities. Pharmacol Ther 2020;215:107628.

21. Chen C, Wang F, Chen P, Jiang J, Cui G, Zhou N, et al. Mortality and Pre-Hospitalization use of Renin-Angiotensin System Inhibitors in Hypertensive COVID-19 Patients. J Am Heart Assoc 2020:e017736.

22. Sanders JM, Monogue ML, Jodlowski TZ and Cutrell JB. Pharmacologic Treatments for Coronavirus Disease 2019 (COVID-19): A Review. JAMA 2020;323:1824-36.

23. Wang M, Cao R, Zhang L, Yang X, Liu J, Xu M, et al. Remdesivir and chloroquine effectively inhibit the recently emerged novel coronavirus (2019-nCoV) in vitro. Cell Res 2020;30:269-71.

24. Gautret P, Lagier JC, Parola P, Hoang VT, Meddeb L, Mailhe M, et al. Hydroxychloroquine and azithromycin as a treatment of COVID-19: results of an open-label non-randomized clinical trial. Int J Antimicrob Agents 2020;56:105949.

25. Yu B, Li C, Chen P, Zhou N, Wang L, Li J, et al. Low dose of hydroxychloroquine reduces fatality of critically ill patients with COVID-19. Sci China Life Sci 2020:1-7.

26. Yu B, Li C, Chen P, Li J, Jiang H and Wang DW. Beneficial effects exerted by hydroxychloroquine in treating COVID-19 patients via protecting multiple organs. Sci China Life Sci 2020:1-4.

27. Arshad S, Kilgore P, Chaudhry ZS, Jacobsen G, Wang DD, Huitsing K, et al. Treatment with hydroxychloroquine, azithromycin, and combination in patients hospitalized with COVID-19. Int J Infect Dis 2020;97:396-403.

28. Geleris J, Sun Y, Platt J, Zucker J, Baldwin M, Hripcsak G, et al. Observational Study of Hydroxychloroquine in Hospitalized Patients with Covid-19. N Engl J Med 2020;382:2411-18.

29. Rosenberg ES, Dufort EM, Udo T, Wilberschied LA, Kumar J, Tesoriero J, et al. Association of Treatment With Hydroxychloroquine or Azithromycin With In-Hospital Mortality in Patients With COVID-19 in New York State. JAMA 2020;323:2493-502.

30. Huang C, Wang Y, Li X, Ren L, Zhao J, Hu Y, et al. Clinical features of patients infected with 2019 novel coronavirus in Wuhan, China. Lancet 2020;395:497-506.

31. Wang D, Hu B, Hu C, Zhu F, Liu X, Zhang J, et al. Clinical Characteristics of 138 Hospitalized Patients With 2019 Novel Coronavirus-Infected Pneumonia in Wuhan, China. JAMA 2020;323:1061-9.

32. Wu Z, McGoogan JM. Characteristics of and Important Lessons From the Coronavirus Disease 2019 (COVID-19) Outbreak in China: Summary of a Report of 72314 Cases From the Chinese Center for Disease Control and Prevention. JAMA 2020; 323: 1239-42.

33. Ruan Q, Yang K, Wang W, Jiang L and Song J. Clinical predictors of mortality due to COVID-19 based on an analysis of data of 150 patients from Wuhan, China. Intensive Care Med 2020;46:846-48.

34. Li C, Jiang J, Wang F, Zhou N, Veronese G, Moslehi JJ, et al. Longitudinal correlation of biomarkers of cardiac injury, inflammation, and coagulation to outcome in hospitalized COVID-19 patients. J Mol Cell Cardiol 2020;147:74-87.

35. Guan WJ, Ni ZY, Hu Y, Liang WH, Ou CQ, He JX, et al. Clinical Characteristics of Coronavirus Disease 2019 in China. N Engl J Med 2020;382:1708-20.

36. Goyal P, Choi JJ, Pinheiro LC, Schenck EJ, Chen R, Jabri A, et al. Clinical Characteristics of Covid-19 in New York City. N Engl J Med 2020;382:2372-74.

37. McGonagle D, Plein S, O’Donnell JS, Sharif K and Bridgewood C. Increased cardiovascular mortality in African Americans with COVID-19. Lancet Respir Med 2020;8:649-51.

38. Inciardi RM, Adamo M, Lupi L, Cani DS, Di Pasquale M, Tomasoni D, et al. Characteristics and outcomes of patients hospitalized for COVID-19 and cardiac disease in Northern Italy. Eur Heart J 2020;41:1821-29.

39. Grasselli G, Zangrillo A, Zanella A, Antonelli M, Cabrini L, Castelli A, et al. Baseline Characteristics and Outcomes of 1591 Patients Infected With SARS-CoV-2 Admitted to ICUs of the Lombardy Region, Italy. JAMA 2020;323:1574-81.

40. Guo T, Fan Y, Chen M, Wu X, Zhang L, He T, et al. Cardiovascular Implications of Fatal Outcomes of Patients With Coronavirus Disease 2019 (COVID-19). JAMA Cardiol 2020;5:1-8.

41. Shi S, Qin M, Shen B, Cai Y, Liu T, Yang F, et al. Association of Cardiac Injury With Mortality in Hospitalized Patients With COVID-19 in Wuhan, China. JAMA Cardiol 2020;5:802-10.

42. Stefanini GG, Montorfano M, Trabattoni D, Andreini D, Ferrante G, Ancona M, et al. ST-Elevation Myocardial Infarction in Patients With COVID-19: Clinical and Angiographic Outcomes. Circulation 2020;141:2113-16.

43. De Filippo O, D’Ascenzo F, Angelini F, Bocchino PP, Conrotto F, Saglietto A, et al. Reduced Rate of Hospital Admissions for ACS during Covid-19 Outbreak in Northern Italy. N Engl J Med 2020;383: 88-9.

44. De Rosa S, Spaccarotella C, Basso C, Calabrò MP, Curcio A, Filardi PP, et al. Reduction of hospitalizations for myocardial infarction in Italy in the COVID-19 era. Eur Heart J 2020;41:2083-88.

45. Clinical characteristics of 113 deceased patients with coronavirus disease 2019: retrospective study. BMJ 2020;368:m1295.

46. Szekely Y, Lichter Y, Taieb P, Banai A, Hochstadt A, Merdler I, et al. Spectrum of Cardiac Manifestations in COVID-19: A Systematic Echocardiographic Study. Circulation 2020;142:342-53. 
47. Petrilli CM, Jones SA, Yang J, Rajagopalan H, O’Donnell L, Chernyak $\mathrm{Y}$, et al. Factors associated with hospital admission and critical illness among 5279 people with coronavirus disease 2019 in New York City: prospective cohort study. BMJ 2020;369:m1966.

48. Huang W, Li C, Wang Z, Wang H, Zhou N, Jiang J, et al. Decreased serum albumin level indicates poor prognosis of COVID-19 patients: hepatic injury analysis from 2,623 hospitalized cases. Sci China Life Sci 2020:1-10.

49. Wichmann D, Sperhake JP, Lütgehetmann M, Steurer S, Edler C, Heinemann A, et al. Autopsy Findings and Venous Thromboembolism in Patients With COVID-19: A Prospective Cohort Study. Ann Intern Med 2020;173:268-77.

50. Ren B, Yan F, Deng Z, Zhang S, Xiao L, Wu M and Cai L. Extremely High Incidence of Lower Extremity Deep Venous Thrombosis in 48 Patients With Severe COVID-19 in Wuhan. Circulation 2020;142:181-83.

51. Zhang L, Feng X, Zhang D, Jiang C, Mei H, Wang J, et al. Deep Vein Thrombosis in Hospitalized Patients With COVID-19 in Wuhan, China: Prevalence, Risk Factors, and Outcome. Circulation 2020;142:114-28.

52. Guzik TJ, Mohiddin SA, Dimarco A, Patel V, Savvatis K, Marelli-Berg FM, et al. COVID-19 and the cardiovascular system: implications for risk assessment, diagnosis, and treatment options. Cardiovasc Res 2020;116:1666-687.

53. Nishiga M, Wang DW, Han Y, Lewis DB and Wu JC. COVID-19 and cardiovascular disease: from basic mechanisms to clinical perspectives. Nat Rev Cardiol 2020;17:543-58.

54. Tang N, Bai H, Chen X, Gong J, Li D and Sun Z. Anticoagulant treatment is associated with decreased mortality in severe coronavirus disease 2019 patients with coagulopathy. J Thromb Haemost 2020;18:1094-99.

55. Paranjpe I, Fuster V, Lala A, Russak AJ, Glicksberg BS, Levin MA, et al. Association of Treatment Dose Anticoagulation With In-Hospital Survival Among Hospitalized Patients With COVID-19. J Am Coll Cardiol 2020;76:122-24.

56. Deng Q, Hu B, Zhang Y, Wang H, Zhou X, Hu W, et al. Suspected myocardial injury in patients with COVID-19: Evidence from front-line clinical observation in Wuhan, China. Int J Cardiol 2020;311:116-21.

57. Inciardi RM, Lupi L, Zaccone G, Italia L, Raffo M, Tomasoni D, et al. Cardiac Involvement in a Patient With Coronavirus Disease 2019 (COVID-19). JAMA Cardiol 2020;5:1-6.
58. $\mathrm{Hu} \mathrm{H}, \mathrm{Ma}$ F, Wei X, Fang Y. Coronavirus fulminant myocarditis saved with glucocorticoid and human immunoglobulin. Eur Heart J 2021;42:206.

59. Xu Z, Shi L, Wang Y, Zhang J, Huang L, Zhang C, et al. Pathological findings of COVID-19 associated with acute respiratory distress syndrome. Lancet Respir Med 2020;8:420-22.

60. Schaller T, Hirschbühl K, Burkhardt K, Braun G, Trepel M, Märkl B and Claus R. Postmortem Examination of Patients With COVID-19. JAMA 2020;323:2518-20.

61. Yu B, Li C, Sun Y, Wang DW. Insulin Treatment Is Associated with Increased Mortality in Patients with COVID-19 and Type 2 Diabetes. Cell Metab 2021;33:65-77.e2.

62. Zhu L, She ZG, Cheng X, Qin JJ, Zhang XJ, Cai J, et al. Association of Blood Glucose Control and Outcomes in Patients with COVID-19 and Pre-existing Type 2 Diabetes. Cell Metab 2020;31:1068-77. e3.

63. Cheng X, Liu YM, Li H, Zhang X, Lei F, Qin JJ, et al. Metformin Is Associated with Higher Incidence of Acidosis, but Not Mortality, in Individuals with COVID-19 and Pre-existing Type 2 Diabetes. Cell Metab 2020;32:537-47. e3.

64. Zheng X, Zhao Y and Yang L. Acute Kidney Injury in COVID-19: The Chinese Experience. Semin Nephrol 2020;40:430-42.

65. Zou X, Chen K, Zou J, Han P, Hao J and Han Z. Single-cell RNA-seq data analysis on the receptor ACE2 expression reveals the potential risk of different human organs vulnerable to $2019-\mathrm{nCoV}$ infection. Front Med 2020;14:185-92.

66. Chong WH and Saha BK. Relationship Between Severe Acute Respiratory Syndrome Coronavirus 2 (SARS-CoV-2) and the Etiology of Acute Kidney Injury (AKI). Am J Med Sci 2020.

67. Zuo H, Li R, Ma F, Jiang J, Miao K, Li H, et al. Temporal echocardiography findings in patients with fulminant myocarditis: beyond ejection fraction decline. Front Med 2020;14:284-92.

68. Zhang XJ, Qin JJ, Cheng X, Shen L, Zhao YC, Yuan Y, et al. In-Hospital Use of Statins Is Associated with a Reduced Risk of Mortality among Individuals with COVID-19. Cell Metab 2020;32:176-87.e4.

How to cite this article: Li C, Wang DW, Zhao C. Cardiovascular involvement in patients with 2019 novel coronavirus disease. J Transl Intern Med 2021; 9: 152-60. 